\title{
Body mass index trajectories in young adulthood predict non- alcoholic fatty liver disease in middle age: The CARDIA cohort study
}

\author{
Lisa B. VanWagner ${ }^{1,2}$ (D) | Sadiya S. Khan ${ }^{2,3}$ | Hongyan Ning ${ }^{2}$ | Juned Siddique ${ }^{2}$ | \\ Cora E. Lewis ${ }^{4}$ | John J. Carr ${ }^{5}$ | Miriam B. Vos ${ }^{6}$ | Elizabeth Speliotes ${ }^{7}$ | \\ Norah A. Terrault $^{8}$ | Mary E. Rinella ${ }^{1}$ | Donald M. Lloyd-Jones ${ }^{2,3}$ | Norrina B. Allen ${ }^{2}$ \\ ${ }^{1}$ Division of Gastroenterology \& Hepatology, Northwestern University Feinberg School of Medicine, Chicago, IL, USA \\ ${ }^{2}$ Department of Preventive Medicine, Northwestern University Feinberg School of Medicine, Chicago, IL, USA \\ ${ }^{3}$ Division of Cardiology, Northwestern University Feinberg School of Medicine, Chicago, IL, USA \\ ${ }^{4}$ Division of Preventive Medicine, University of Alabama at Birmingham, Birmingham, AL, USA \\ ${ }^{5}$ Department of Radiology, Vanderbilt University, Nashville, TN, USA \\ ${ }^{6}$ Division of Gastroenterology, Department of Pediatrics, Emory University, Atlanta, GA, USA \\ ${ }^{7}$ Division of Gastroenterology, University of Michigan, Ann Arbor, MI, USA \\ ${ }^{8}$ Division of Gastroenterology \& Hepatology, University of California at San Francisco, San Francisco, CA, USA
}

\section{Correspondence}

Lisa B. Van Wagner, MD MSc, Northwestern

University, Departments of Medicine-

Gastroenterology \& Hepatology and

Preventive Medicine, Chicago, IL, USA.

Email: Ivw@northwestern.edu

\section{Funding information}

The CARDIA Study is supported by contracts HHSN268201300025C,

HHSN268201300026C,

HHSN268201300027C,

HHSN268201300028C,

HHSN268201300029C and

HHSN268200900041C from the National

Heart, Lung, and Blood Institute (NHLBI),

the Intramural Research Program of the

National Institute on Aging (NIA) and an intra-

agency agreement between NIA and NHLBI

(AG0005). Dr. VanWagner is supported by the

National Center for Advancing Translational

Sciences (KL2TR001424). Dr. Carr is

supported by the NIH (R01-HL-098445).

Handling Editor: Luca Valenti

\section{Abstract}

Background \& Aims: Non-alcoholic fatty liver disease is an epidemic. Identifying modifiable risk factors for non-alcoholic fatty liver disease development is essential to design effective prevention programmes. We tested whether 25 -year patterns of body mass index change are associated with midlife non-alcoholic fatty liver disease.

Methods: In all, 4423 participants from Coronary Artery Risk Development in Young Adults, a prospective population-based biracial cohort (age 18-30), underwent body mass index measurement at baseline (1985-1986) and 3 or more times over 25 years. At Year 25, 3115 had liver fat assessed by non-contrast computed tomography. Nonalcoholic fatty liver disease was defined as liver attenuation $\leq 40$ Hounsfield Units after exclusions. Latent mixture modelling identified 25 -year trajectories in body mass index per cent change $(\% \Delta)$ from baseline.

Results: We identified four distinct trajectories of BMI\% $\%$ : stable $(26.2 \%$ of cohort, 25 -year BMI $\% \Delta=3.1 \%$ ), moderate increase $(46.0 \%, \mathrm{BMI} \% \Delta=21.7 \%)$, high increase (20.9\%, BMI\% $\Delta=41.9 \%)$ and extreme increase (6.9\%, BMI\% $\Delta=65.9 \%)$. Y25 nonalcoholic fatty liver disease prevalence was higher in groups with greater $\mathrm{BMI} \% \Delta$ : $4.1 \%, 9.3 \%, 13.0 \%$, and $17.6 \%$, respectively ( $P$-trend $<.0001)$. In multivariable analyses, participants with increasing $\mathrm{BMI} \% \Delta$ had increasingly greater odds of non-alcoholic fatty liver disease compared to the stable group: OR: 3.35 (95\% Cl: 2.07-5.42), 7.80 (4.60-13.23) and 12.68 (6.68-24.09) for moderate, high and extreme body mass index

Abbreviations: $\% \Delta$, per cent change; BMI, body mass index; CARDIA, Coronary Artery Risk Development in Young Adults Study; CI, confidence interval; CT, computed tomography; EU, exercise units; HDL-C, high-density lipoprotein cholesterol; HOMA-IR, homeostatic model assessment of insulin resistance; HU, Hounsfield units; LA, liver attenuation; MR, magnetic resonance imaging; NAFLD, non-alcoholic fatty liver disease; NASH, non-alcoholic steatohepatitis; OR, odds ratio; SBP, systolic blood pressure; WC, waist circumference; WHR, waist-to-hip circumference ratio. 
increase, respectively. Associations were only moderately attenuated when adjusted for baseline or $\mathrm{Y} 25$ body mass index.

Conclusions: Trajectories of weight gain during young adulthood are associated with greater non-alcoholic fatty liver disease prevalence in midlife independent of metabolic covariates and baseline or concurrent body mass index highlighting the importance of weight maintenance throughout adulthood as a target for primary non-alcoholic fatty liver disease prevention.

KEYWORDS

NAFLD, NASH, obesity, prevention

\section{1 | INTRODUCTION}

Non-alcoholic fatty liver disease (NAFLD) is the most common cause of chronic liver disease in both children and adults, affecting approximately $30 \%$ of the population. ${ }^{1,2}$ NAFLD represents a spectrum of liver conditions that are associated with differential risk for the development of cirrhosis, ${ }^{3}$ liver cancer, ${ }^{4}$ need for liver transplantation ${ }^{5}$ and cardiovascular disease. ${ }^{6}$ In addition, mortality is up to $69 \%$ higher among persons with NAFLD compared to persons without NAFLD. ${ }^{7}$ Thus, improving our understanding of how to prevent NAFLD has the potential to save millions of lives worldwide.

Non-alcoholic fatty liver disease is the hepatic manifestation of the metabolic syndrome, and being overweight or obese is associated with greater odds of having NAFLD. ${ }^{3,8}$ Weight gain throughout adulthood, as opposed to weight at a single time point, has been reported to be a risk factor for various lifestyle-related diseases including diabetes ${ }^{9}$ and cardiovascular diseases. ${ }^{10-12}$ However, little is known about objective longterm weight patterns and NAFLD prevalence, especially among a young, contemporary and racially diverse population. ${ }^{13,14}$ It remains unclear whether long-term patterns of weight gain through younger adulthood, regardless of concurrent body mass index (BMI) or weight category (e.g. normal weight, overweight or obese), adversely influence NAFLD prevalence in middle age. Recognition of patterns of weight change over time may allow earlier identification of patients who are at risk for developing NAFLD and optimize strategies aimed at primary NAFLD prevention.

We therefore sought to examine the association of BMI trajectories (e.g. patterns of change over time) among young adults (age 18-30 years) over 25 years with NAFLD defined by computed tomography (CT) in middle age (43-55 years). We hypothesized that multiple different trajectories of BMI change exist throughout adulthood, and that those groups with greater BMI increase throughout adulthood would have a higher prevalence of NAFLD in middle age, independent of baseline or concurrent BMI.

\section{2 | METHODS}

\section{1 | Study sample}

The Coronary Artery Risk Development in Young Adults (CARDIA) study is an on-going longitudinal cohort study of 5115 biracial men

\section{Key points}

- NAFLD is an epidemic associated with increased liver and non-liver-related morbidity and mortality.

- Overweight and obesity weight categories are highly associated with NAFLD and early identification of modifiable risk factors is an important strategy to decrease the NAFLD burden.

- Increasing patterns of weight change throughout adulthood are associated with differential risk for NAFLD in midlife independent of starting or concurrent weight or weight category.

- Strategies aimed at weight maintenance through young adulthood, rather than weight loss attempts later, are likely to be most successful at preventing NAFLD.

and women from 4 metropolitan populations. Participants were 1830 years of age at enrolment (1985-1986, exam year 0). Recruitment was balanced within each centre by sex, age, race and education. Participants have been followed at 9 examinations for more than 30 years with collection of detailed clinical data, including non-contrast CT measurement of liver fat at year 25 (2010-2011). Retention rates among survivors for the in-person examinations have been high (Y2, 90\%; Y5, 86\%; Y7, 81\%; Y10, 77\%; Y15, 74\%; Y20, 72\%; Y25, 72\%; $\mathrm{Y} 30,71 \%)$ and $>90 \%$ of initial participants have maintained contact over time. ${ }^{15}$ Participants provided written informed consent at each examination, and institutional review boards from each field centre (University of Alabama at Birmingham, Birmingham, Alabama; Northwestern University, Chicago, Illinois; University of Minnesota, Minneapolis, Minnesota; and Kaiser Permanente, Oakland, California) approved the study annually.

Trajectories of per cent change $(\% \Delta)$ in BMI relative to baseline were modelled among all 4423 participants with BMI measured at baseline and at 3 or more follow-up examinations. BMI values at examinations at which the participant was pregnant $(n=266)$ were excluded. Of the 3430 participants with repeat BMI measures at Y25, 3115 had liver fat assessed. We excluded those with self-reported 
cirrhosis or viral hepatitis $(n=54)$, risk factors for chronic liver disease (e.g. intravenous drug use, $\mathrm{n}=81$ ) or causes of secondary hepatic steatosis: alcohol consumption $\geq 7$ drinks/wk in women and $\geq 14$ drinks/ wk in men $(n=280),{ }^{16}$ human immunodeficiency virus $(n=23)$, and medications known to cause hepatic steatosis (e.g. valproic acid, methotrexate, tamoxifen, steroids, amiodarone) $(n=27)$. The remaining 2650 formed the NAFLD-eligible sample population (Figure 1).

\section{2 | Measurements}

Standardized protocols for data collection were used across study centres and have previously been described. ${ }^{15,17}$ Weight and height were measured with participants wearing light clothes and no shoes at each of the 8 examinations. Body weight was measured to the nearest $0.2 \mathrm{~kg}$ with a calibrated balance-beam scale. Height was measured with a vertical ruler to the nearest $0.5 \mathrm{~cm}$. BMI was calculated as weight in kilograms divided by height in meters squared. Waist circumference (WC) was measured in duplicate to the nearest $0.5 \mathrm{~cm}$ around the minimal abdominal girth identified laterally midway between the iliac crest and the lowest portion of the rib cage and anteriorly midway between the xiphoid process and the umbilicus parallel to the floor. Hip circumference was measured in duplicate to the nearest $0.5 \mathrm{~cm}$ at the level of the pubis symphysis anteriorly and posteriorly at the level of the maximal protrusion of the gluteal muscles. Participants were asked to fast for 12 hours and to avoid smoking and heavy physical activity for 2 hours before each examination. Overweight was defined as BMI $25-29.9 \mathrm{~kg} / \mathrm{m}^{2}$, class I obesity as BMI $30-34.9 \mathrm{~kg} / \mathrm{m}^{2}$, class II as BMI $35-39.9 \mathrm{~kg} / \mathrm{m}^{2}$ and class III obesity as $\mathrm{BMI} \geq 40 \mathrm{~kg} / \mathrm{m}^{2}$. The metabolic syndrome was defined according

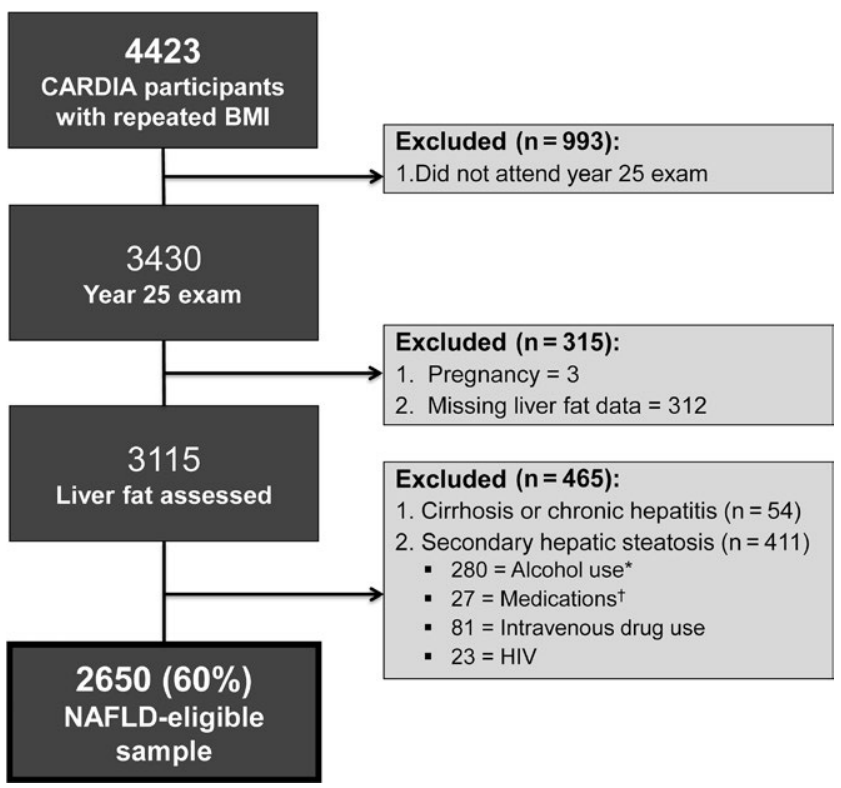

FIGURE 1 Study population. Abbreviations: CT, computed tomography; HIV, human immunodeficiency virus. *Alcohol use was defined as $\geq 7$ drinks/wk in women, $\geq 14$ drinks/wk in men. ${ }^{\dagger}$ Medications = valproic acid, methotrexate, tamoxifen, steroids and amiodarone to Adult Treatment Panel III criteria. ${ }^{18}$ To quantify physical activity (reported as exercise units [EU]), the CARDIA physical activity history questionnaire was used, which was an interviewer-based self-report of duration and intensity of participation in 13 categories of exercise over the previous 12 months. ${ }^{17}$ As a reference, $300 \mathrm{EU}$ approximates 150 minutes of moderate-intensity activity per week or 30 minutes of moderate-intensity activity 5 days per week. ${ }^{17}$ All CARDIA protocols are publicly available at: http://www.cardia.dopm.uab.edu.

The CT protocol included the heart and abdomen using a noncontrast CT scan performed using GE (GE 750HD 64 and GE LightSpeed VCT 64 Birmingham and Oakland Centers, respectively; GE Healthcare, Waukesha, WI, USA) or Siemens (Sensation 64, Chicago and Minneapolis Centers; Siemens Medical Solutions, Erlangen, Germany) multi-detector CT scanners and has been described previously. ${ }^{19}$ Quality control and image analysis were performed at a core reading centre (Wake Forest University Health Sciences, WinstonSalem, NC).

The primary outcome was NAFLD at Y25 defined as liver attenuation (LA) $\leq 40$ Hounsfield Units (equivalent to moderate-severe fat) ${ }^{20}$ after exclusion of other causes of liver fat (Figure 1). ${ }^{19}$ LA was measured in the right lobe of the liver and was reported as the average of 9 measurements on 3 CT slices using circular regions of interest of $2.6 \mathrm{~cm}^{2}$. The intraclass correlation coefficient between different readers on a random selected sample of 156 participants was 0.975 for LA, indicating high reproducibility of CT measured LA.

\section{3 | Statistical analysis}

Trajectories in BMI, WC and waist-to-hip ratio (WHR) \% $\Delta$ were modelled among all participants ( $N=4423$ ) using data from each examination attended. The $\% \Delta$ was modelled rather than absolute values of increase since $\% \Delta$ more appropriately represents baseline and relative change values. We used latent class models to identify subgroups that share a similar trajectory in BMI, WC or WHR $\% \Delta .{ }^{21}$ The optimal number of trajectory classes was determined using the Bayesian information criterion such that no group included $<5 \%$ of the population. To estimate the association of trajectory group with prevalent NAFLD, trajectory group membership was included as an independent variable in a logistic regression model examining predictors of Y25 NAFLD. To account for the uncertainty in BMI $\% \Delta$ trajectory group assignment, we calculated the posterior predicted probability for each individual of being a member in each of the classes. ${ }^{22}$ Participants were assigned to the trajectory group for which they had the greatest posterior predictive probability. Models were sequentially adjusted a priori for potential confounders including demographics (baseline age, sex, race, education, centre), cumulative burden of metabolic risk factors (cumulative systolic blood pressure [SBP], number of visits with blood pressure medications, cumulative triglycerides, cumulative years of diabetes, pack-years of cigarette smoking exposure, cumulative alcohol use [drinks/d], cumulative physical activity [EU per year]), and BMI at baseline (YO) or at the time of NAFLD assessment (Y25). Cumulative SBP, alcoholic beverages, physical activity and triglycerides were calculated by summing the product of the 
average SBP (or triglycerides or alcohol or physical activity) and the time interval (in years) between 2 consecutive examinations over the 25 years. Interaction terms were assessed between trajectory group membership and race and sex. We compared the predictive utility of $\mathrm{BMI} \% \Delta$ trajectory group compared with other adiposity measures (WC or WHR) using the $\mathrm{C}$ statistic derived from the logistic regression models. Missing data were excluded from analyses. All variables analysed had $<1 \%$ missing data. All analyses were completed using SAS software version 9.4 (SAS Institute Inc., Chicago, IL, USA). Two-sided $P<.05$ was considered statistically significant.

\section{3 | RESULTS}

\section{1 | Fat distribution and metabolic characteristics}

Four discrete trajectories in $\mathrm{BMI} \% \Delta$ from young adulthood to middle age were identified (Figure 2). In general, BMI increased over time in the majority of participants: only $26.2 \%$ of the cohort ( $n=1159$ ) maintained stable BMI $\% \Delta$ throughout follow-up (stable; BMI $\% \Delta=3.1 \% \pm 9.1 \%$ [mean \pm SD] $) ; 46.0 \%(n=2035)$ had moderate $\mathrm{BMI}$ increase (moderate increase; $\mathrm{BMI} \% \Delta=21.7 \% \pm 9.7 \%$ ), 20.9\% ( $n=923$ ) had high BMI increase (high increase; BMI $\% \Delta=41.8 \% \pm 13.8 \%)$ and $6.9 \%(n=306)$ had an extreme BMI increase (extreme increase; BMI $\% \Delta=65.9 \% \pm 19.3 \%$ ), with a notable early rapid $\% \Delta$ in BMI. The observed BMI \% $\Delta$ corresponded to an average weight gain over 25 years of $1.9 \pm 8.0,15.3 \pm 7.5,29.0 \pm 11.0$ and $43.8 \pm 14.1 \mathrm{~kg}$ in the stable, moderate, high and extreme increase groups, respectively.

Participant characteristics at the baseline examination according to $\mathrm{BMI} \% \Delta$ trajectory group are presented in Table 1 . Individuals with a higher BMI \% $\Delta$ trajectory were younger and more likely to be female, black and have lower education than the stable group. At Y25, groups with greater increases in BMI had a higher prevalence of components of the metabolic syndrome manifested by higher diabetes prevalence, fasting glucose, HOMA-IR and triglycerides and lower HDL levels (Table 2).

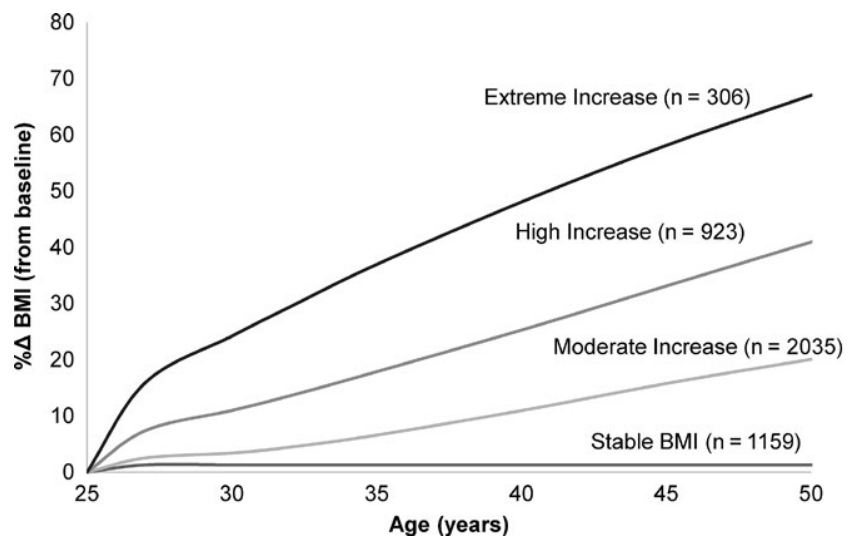

FIGURE 2 Per cent Change (\% $\%$ ) Body Mass Index (BMI) Trajectories by Age in the Coronary Artery Risk Development in Young Adults (CARDIA) Study. Total N = 4423
At baseline, the majority of participants were normal weight (>62\%) and the extreme increase group had the smallest proportion of obesity (5.6\%, Table S1). However, over time, the proportion of overweight/obesity increased substantially, particularly among groups with substantial $\% \Delta$ in BMI. Mean BMI at Y25 was $25.9 \pm 5.7,29.5 \pm 5.8$, $34.3 \pm 7.2$ and $38.9 \pm 7.3 \mathrm{~kg} / \mathrm{m}^{2}$ in the stable, moderate, high increase and extreme increase groups, respectively (Table S1).

\section{$3.2 \mid \% \Delta \mathrm{BMI}$ and NAFLD}

Non-alcoholic fatty liver disease prevalence at Y25 was higher with increasing BMI change group and was present in $4.1 \%(n=38), 9.3 \%$ $(n=146), 13.0 \%(n=93)$ and $17.6 \%(n=39)$ in the stable, moderate increase, high increase and extreme increase groups, respectively $(P$ trend $<.0001$, Figure S1A). In comparison with individuals in the stable group, those in trajectory groups with patterns of increasingly severe BMI $\% \Delta$ had progressively greater odds of having NAFLD even when adjusted for demographics, education and cardiovascular risk factors (ORs 3.35 [95\% Cl: 2.07-5.43], 7.80 [4.60-13.23] and 12.68 [6.6824.09]) for moderate, high and extreme increase groups, respectively, Table 3). These associations were only moderately attenuated when adjusted for baseline BMI (Table 3). Associations were attenuated more substantially, but remained statistically significant when adjusted for Y25 BMI (Table 3).

Figure 3 demonstrates Y25 NAFLD prevalence stratified by weight category at baseline according to BMI $\% \Delta$ trajectory group. Within each trajectory group, NAFLD prevalence increased with increasing weight category group. However, participants with class I obesity who maintained a stable BMI over time were significantly less likely to have prevalent NAFLD at Y25 compared with those who were normal weight at baseline, but who had an extreme increase in $\mathrm{BMI} \% \Delta$ over time (e.g. 6.5\% vs $17.9 \%, P<.0001$ ). Associations were similar, though less pronounced, for participants with baseline class II (15.7\%) and class III (16.2\%) obesity who maintained stable weight compared with those who were normal weight at baseline with extreme BMI increase over time $(P<.05$ for both, respectively).

We identified a significant interaction between $\mathrm{BMI} \% \Delta$ trajectory group with both race and sex on the odds of Y25 NAFLD. Despite this statistical interaction, among all race-sex groups the odds of NAFLD increased with increasing $\mathrm{BMI} \% \Delta$ trajectories and whereas we observed different magnitudes of association across race-sex groups, the direction of the association did not differ. The prevalence of NAFLD was low $(<5 \%)$ in several subgroups, particularly in white females and black males with stable BMI (Table S2); thus, the point estimates for odds of NAFLD by BMI \% $\Delta$ trajectory group had poor precision in analyses stratified by race and sex (e.g. 95\% Cl: 14.8-427.4, Table S3).

\section{3 | $\% \Delta$ Waist circumference and waist-to-hip ratio}

Overall patterns of change throughout adulthood were similar when we examined the trajectories of $\% \Delta$ in WC or WHR separately (Figure S2). Changes in WC and WHR trajectory groups were highly to moderately correlated with BMI \% groups (Spearman $r=.82$ for WC 
TAB LE 1 Baseline characteristics at year 0 by body mass index per cent change $(\% \Delta)$ trajectory group

\begin{tabular}{|c|c|c|c|c|c|}
\hline $\begin{array}{l}\text { Demographic } \\
\text { characteristics }^{\text {a }}\end{array}$ & Stable $N=1159$ & $\begin{array}{l}\text { Moderate increase } \\
\mathrm{N}=2035\end{array}$ & High increase $\mathrm{N}=923$ & $\begin{array}{l}\text { Extreme increase } \\
N=306\end{array}$ & $P$ value $^{b}$ \\
\hline Age, years & $26.1(3.2)$ & $25.5(3.4)$ & $23.4(3.5)$ & $21.6(3.2)$ & $<.0001$ \\
\hline Blacks & $440(38.0)$ & $949(46.6)$ & $576(62.4)$ & $214(69.9)$ & \multirow[t]{2}{*}{$<.0001$} \\
\hline Whites & $719(62.0)$ & $1086(53.4)$ & $347(37.6)$ & $92(30.1)$ & \\
\hline Women & $605(52.2)$ & $1058(52.0)$ & $546(59.2)$ & $223(72.9)$ & \multirow[t]{2}{*}{$<.0001$} \\
\hline Men & $554(47.8)$ & $997(48.0)$ & 377 (40.9) & $83(27.1)$ & \\
\hline Education, years & $14.4(2.4)$ & $14.0(2.3)$ & $13.3(1.9)$ & $12.8(1.7)$ & $<.0001$ \\
\hline Current smoker & $338(29.3)$ & $585(28.9)$ & $262(28.6)$ & $83(27.3)$ & .08 \\
\hline Alcohol drinker & $780(67.6)$ & $1272(62.8)$ & $493(53.7)$ & $144(47.1)$ & $<.0001$ \\
\hline Weight, kg & $73.8(18.3)$ & $70.8(15.4)$ & $69.8(15.5)$ & $66.8(13.2)$ & $<.0001$ \\
\hline Height, $\mathrm{cm}$ & $171.2(9.5)$ & $170.6(9.4)$ & $169.6(9.7)$ & $167.7(8.9)$ & .001 \\
\hline $\mathrm{BMI}, \mathrm{kg} / \mathrm{m}^{2}$ & $25.2(5.9)$ & $24.3(4.7)$ & $24.3(4.7)$ & $23.8(4.1)$ & $<.0001$ \\
\hline Waist circumference, $\mathrm{cm}$ & $79.9(13.2)$ & $77.8(10.8)$ & $76.2(10.6)$ & $74.1(8.6)$ & $<.0001$ \\
\hline Waist-hip ratio & $0.79(0.07)$ & $0.78(0.07)$ & $0.76(0.06)$ & $0.75(0.07)$ & $<.0001$ \\
\hline Systolic BP, mm Hg & $111.1(11.2)$ & $110.5(10.9)$ & $109.6(10.4)$ & $109.0(11.6)$ & .001 \\
\hline Diastolic BP, mm Hg & $69.6(9.9)$ & $68.8(9.6)$ & $67.6(9.3)$ & $66.6(9.2)$ & $<.0001$ \\
\hline Hypertension & $62(5.4)$ & $73(3.6)$ & $31(3.4)$ & $11(3.6)$ & .06 \\
\hline Fasting glucose, $\mathrm{mg} / \mathrm{dL}$ & $84.5(21.2)$ & $82.3(14.3)$ & $80.7(8.4)$ & $80.2(9.3)$ & $<.0001$ \\
\hline
\end{tabular}

$\mathrm{SD}$, standard deviation; n, number; BMI, body mass index; BP, blood pressure; HDL, high-density lipoprotein.

All results are presented as mean (SD) or $\mathrm{n}(\%)$.

${ }^{a}$ All variables have $<1 \%$ missing.

${ }^{\mathrm{b}} \mathrm{Chi}$-square test for categorical variables and ANOVA for continuous variables.

and .39 for WHR, $P<.0001$ for both). Both WC and WHR \% $\Delta$ were similarly associated in a dose-response fashion with NAFLD at Y25 (Figures S1B and C). As expected, associations with measures of central adiposity $\% \Delta$ and prevalent NAFLD demonstrated similar trends as those models that used BMI \% $\Delta$ (Tables S4 and S5). In the multivariable adjusted model, WC $\% \Delta$ discriminated prevalent NAFLD comparably to $\mathrm{BMI} \% \Delta$ (C statistic, 0.784 for WC and 0.780 for $\mathrm{BMI}$ ) and slightly better than WHR \% (C statistic, 0.754).

\section{4 | DISCUSSION}

In a large, population-based, prospective study of biracial adults followed for 25 years, we identified four trajectories of BMI change that were significantly associated with prevalent NAFLD in midlife. We found that those groups with greater BMI increase from young adulthood to middle age have the greatest odds of having NAFLD in midlife, regardless of demographics, the cumulative burden of clinical covariates and weight status at baseline or concurrently. Indeed, those who had baseline obesity and had major BMI increases through young adulthood had NAFLD prevalence of $28 \%-40 \%$; conversely, those who had baseline obesity but maintained stable BMI had NAFLD prevalence of only $16 \%$. These findings highlight the importance of weight maintenance throughout young adulthood, regardless of baseline weight, as a critical target for the primary prevention of NAFLD.

The current study provides unique insights into long-term patterns of change in BMI during early adulthood. Importantly, participants at baseline had similar mean BMI values, yet their patterns over time diverged markedly. In CARDIA, a large proportion of participants developed incident obesity over time with increases in BMI in all groups to varying degrees. We identified heterogeneous patterns of BMI change as separate trajectory groups, thus providing increased understanding of lifetime trends in weight. Knowledge of these trajectories throughout adulthood may allow us to potentially attenuate the NAFLD 
TABLE 2 Follow-up characteristics at year 25 by body mass index per cent change $(\% \Delta)$ trajectory group

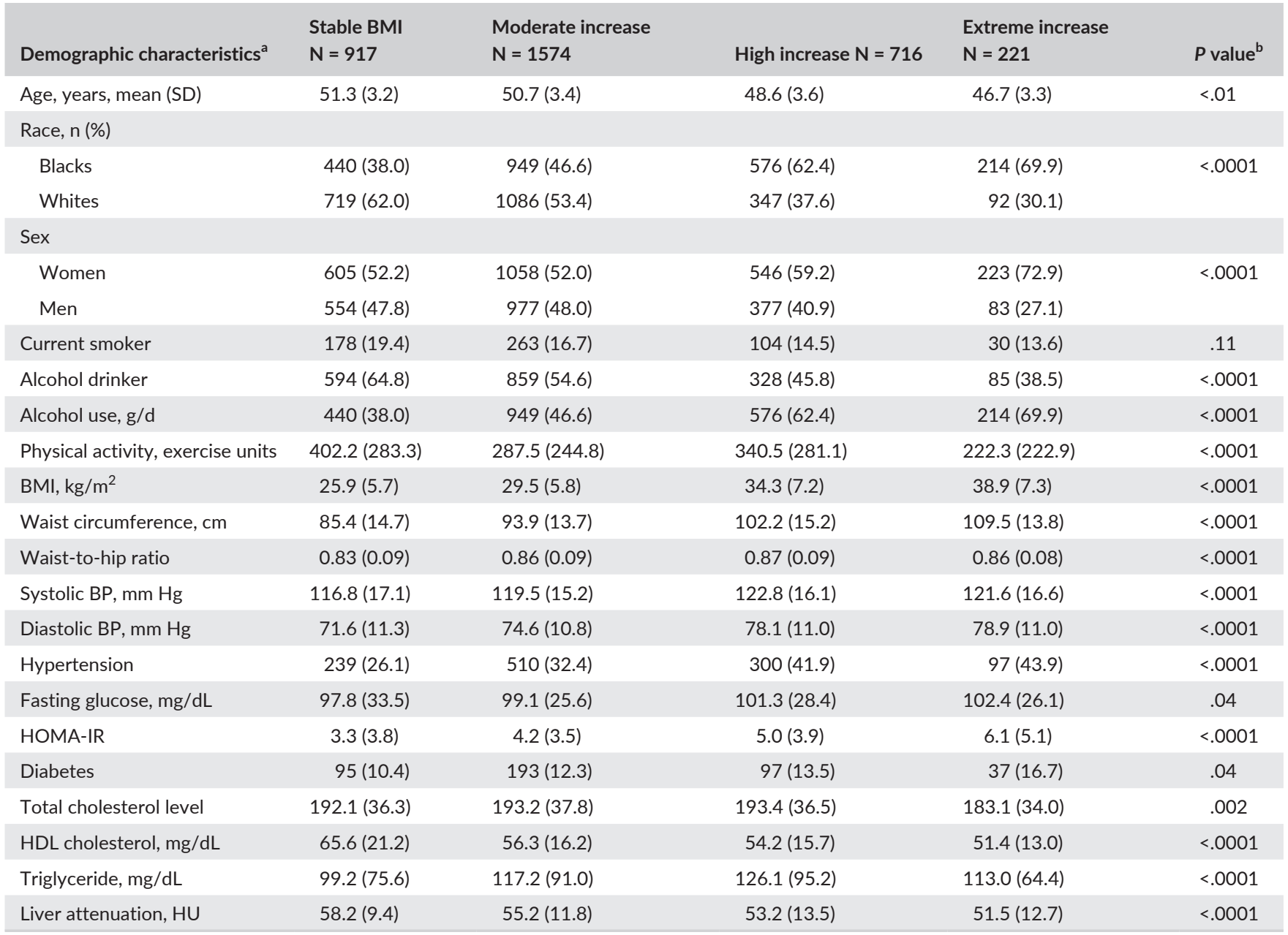

SD, standard deviation; n, number; BMI, body mass index; BP, blood pressure; HOMA-IR, Homeostatic model assessment for insulin resistance; HDL, highdensity lipoprotein; HU, Hounsfield units.

All results are presented as mean (SD) or $\mathrm{n}(\%)$.

${ }^{a}$ All variables have $<1 \%$ missing.

${ }^{b}$ Chi-square test for categorical variables and ANOVA for continuous variables.

TABLE 3 Odds ratios for the association of BMI \% $\Delta$ trajectory group with prevalent NAFLD in middle age in CARDIA

\begin{tabular}{|c|c|c|c|c|c|c|c|c|}
\hline \multirow[b]{2}{*}{ BMI \% $\%$ group } & \multicolumn{2}{|l|}{$\begin{array}{l}\text { Unadjusted } \\
\mathrm{N}=2615\end{array}$} & \multicolumn{2}{|l|}{$\begin{array}{l}\text { Base model }^{\mathrm{a}} \\
\mathrm{N}=2615\end{array}$} & \multicolumn{2}{|l|}{$\begin{array}{l}\text { Base model + YO BMI } \\
\mathrm{N}=2615\end{array}$} & \multicolumn{2}{|c|}{$\begin{array}{l}\text { Base model + Y25 BMI } \\
\mathrm{N}=2611\end{array}$} \\
\hline & OR $(95 \% \mathrm{Cl})$ & $P$ value & OR $(95 \% \mathrm{Cl})$ & $P$ value & OR $(95 \% \mathrm{Cl})$ & $P$ value & OR $(95 \% \mathrm{Cl})$ & $P$ value \\
\hline Stable & Reference & & & & & & & \\
\hline Moderate increase & $2.65(1.71-4.09)$ & .005 & $3.35(2.07-5.43)$ & .04 & $4.40(2.66-7.31)$ & .03 & $2.56(1.55-4.21)$ & .69 \\
\hline High increase & $4.27(2.71-6.73)$ & .0002 & $7.80(4.60-13.23)$ & $<.0001$ & $11.38(6.51-19.89)$ & $<.0001$ & $3.73(2.13-6.52)$ & .001 \\
\hline
\end{tabular}

BMI, body mass index; NAFLD, non-alcoholic fatty liver disease; OR, odds ratio; $\mathrm{Cl}$, confidence interval.

Results presented as Odds Ratio (95\% Confidence interval).

NAFLD = liver attenuation $\leq 40 \mathrm{HU}$ after exclusion for secondary causes of liver fat.

${ }^{a}$ Base model adjusted for baseline age, sex, race, education, centre, cumulative systolic blood pressure (mm Hg-years), number of years with blood pressure medications, cumulative triglycerides (mg/dL-years), cumulative years with diabetes, cumulative alcohol use (drinks/d), physical activity level (exercise units-year) and pack-years of cigarette smoking exposure. 


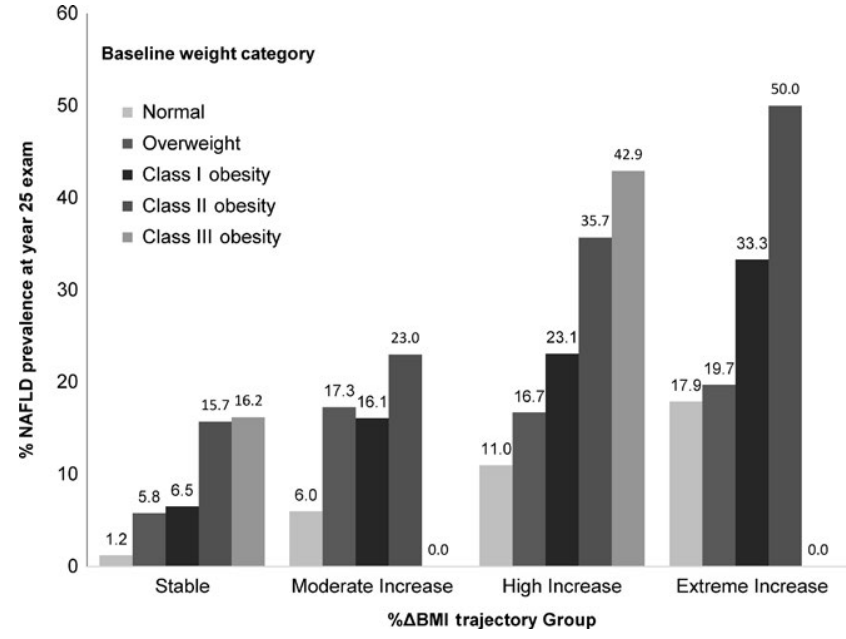

FIGURE 3 Year 25 non-alcoholic fatty liver disease (NAFLD) Prevalence Stratified by Baseline Weight Category and Per cent Change $(\% \Delta)$ in BMI Trajectory Group (Total N at Year 25 exam $=2650$ ). Class I obesity was defined as body mass index (BMI) $30-34.9 \mathrm{~kg} / \mathrm{m}^{2}$, class II obesity as BMI $35-39.9 \mathrm{~kg} / \mathrm{m}^{2}$, class III obesity as $\mathrm{BMI} \geq 30 \mathrm{~kg} / \mathrm{m}^{2}$, overweight as BMI $25-29.9 \mathrm{~kg} / \mathrm{m}^{2}$ and normal weight as $\mathrm{BMI}<25 \mathrm{~kg} / \mathrm{m}^{2}$

epidemic, highlighting the period of transition from young adulthood to middle age as a prime target for health promotion, primordial prevention and long-term disease prevention.

The association between adult weight gain and NAFLD was independent of multiple metabolic risk factors associated with histological progression of NAFLD. Body weight gain in earlier adulthood (age 2540 years) has been associated with increased markers of insulin resistance compared with later adulthood weight gain (age $>40$ years). ${ }^{23}$ In a cross-sectional study of 1119 Chinese participants (mean age 47), earlier increases in insulin resistance mediated the relationship between adult weight gain and NAFLD. ${ }^{13}$ In addition, BMI increase in earlier adulthood is more strongly associated with unfavourable levels of obesity biomarkers (e.g. adiponectin) and markers of liver damage (e.g. gammaglutamyl transferase), than BMI gain in later adulthood. ${ }^{23}$ Therefore, the increased NAFLD prevalence in midlife among adults with a steep, early increase in BMI (as was observed in our extreme increase trajectory group) may in fact be mediated by the systemic influences of visceral adiposity. This is supported by the finding that increased WC, a marker of visceral adiposity, was highly predictive of prevalent NAFLD.

We observed significant differences in the strength of the association of BMI trajectory and NAFLD prevalence by race and sex. NAFLD prevalence was highest in white men followed by white women, black men and black women, whereas obesity prevalence was highest among black women and black men. There are significant differences in NAFLD prevalence between racial-ethnic groups with a higher risk of severe disease in Hispanics and Whites, and a surprisingly low risk in African Americans, for reasons that are not entirely known. ${ }^{8,24}$ Future studies are needed to assess the potential impact of adult weight gain among various racial-ethnic populations who may be at differential risk for NAFLD despite a high prevalence of obesity.

\section{1 | Strengths and limitations}

Our study has several strengths. The CARDIA study is wellpositioned to examine longitudinal trends in BMI and its relationship to NAFLD since the cohort began at the start of the obesity epidemic (1985-86), prior to the onset of the NAFLD epidemic, and involves a biracial population with a wealth of rigorously measured covariates. In addition to our BMI findings, we saw similar trajectory patterns with measures of central fat distribution (WC, WHR) with greater NAFLD prevalence associated with greatest increases in these measures, independent of cumulative comorbidities. Finally, we applied innovative statistical methods to examine patterns of changes in adiposity in a large, well-characterized cohort of black and white Americans.

Several limitations should also be considered when interpreting our study results. NAFLD prevalence in CARDIA is somewhat lower than what has been reported in other US cohorts. Our NAFLD outcome was measured by LA on non-contrast CT which is a relatively insensitive tool for liver fat assessment compared to magnetic resonance imaging (e.g. MR-PDFF or spectroscopy), which are not available in CARDIA. ${ }^{20,25}$ We chose our LA cut-off based on previous studies correlating LA with histology, which showed excellent specificity but lower sensitivity for the detection of NAFLD. ${ }^{20}$ Maintaining high specificity minimizes the impact of measurement bias; however, our LA cut-off could not detect lesser degrees of pathologic steatosis between 5\% and 30\%. Second, CARDIA included a biracial cohort of adults and we observed a lower NAFLD prevalence in blacks compared to whites consistent with other studies. ${ }^{24}$ Our cohort also did not ask about Hispanic ethnicity or obtain data on genetic polymorphisms including patatin-like phospholipase domain containing protein 3 (PNPLA3), which partially explain racial differences in NAFLD. Thus, our findings cannot be generalized to other ethnic groups. Contemporaneous or baseline laboratory data on hepatic function are not available in CARDIA. However, there is no laboratory test for NAFLD and serum aminotransferases are often normal despite the presence of liver injury. ${ }^{26}$ NAFLD was also not assessed in CARDIA prior to the Y25 follow-up examination and thus, we do not know when during adulthood NAFLD may have developed. However, since NAFLD is primarily an asymptomatic disease, detection in midlife mirrors clinical practice when NAFLD is commonly incidentally found on imaging performed for other reasons. ${ }^{27}$ It is also possible that some CARDIA participants had undiagnosed NAFLD at the baseline examination. However, over $62 \%$ of NAFLD participants were normal weight at baseline. Finally, viral hepatitis status was obtained by self-report, raising the possibility of undiagnosed hepatitis, particularly Hepatitis C, within our cohort.

\subsection{Clinical and public health implications}

Weight loss has been associated with biochemical, ${ }^{28}$ radiographic ${ }^{29,30}$ and histological ${ }^{31,32}$ improvements in NAFLD, but initial weight loss may be a challenge and maintaining lower weight after weight loss is difficult over time. ${ }^{33}$ Weight loss and physical activity are the recommended treatments for NAFLD. ${ }^{16}$ However, efficacy of lifestyle intervention is poor due to a lack of patient adherence, programmes 
designed specifically for patients with liver disease, and financial support from payers to sustain these programmes long-term. ${ }^{16,34}$ Finally, lifestyle interventions in early adult life are more likely to be successful than interventions attempted later in life once lifestyle habits and diseases have further progressed. ${ }^{35}$ In addition to weight loss as a treatment for obesity (e.g. secondary prevention), our data suggest that primary prevention strategies aimed at weight maintenance through young adulthood are likely to have a significant impact at preventing NAFLD and its consequences. Thus, maintaining weight throughout adulthood regardless of starting point (e.g. normal weight, overweight or obese) may reduce NAFLD risk in middle age.

\section{5 | CONCLUSION}

Our findings imply that the trajectory of BMI change throughout early adulthood to midlife - independent of baseline and concurrent BMI provides additional information about the risk of developing NAFLD. These associations were independent of key comorbidities and metabolic risk factors. This novel characterization of change in BMI trajectories across a critical period for significant weight gain highlights young adulthood as an important target for behaviour and lifestyle interventions for primordial prevention of NAFLD. Prevention programmes that target weight maintenance in early adulthood, regardless of starting weight or weight category, may be more effective for NAFLD prevention than programmes that target weight loss after the disease has developed in later life.

\section{ACKNOWLEDGEMENTS}

The authors thank the participants of the CARDIA study for their long-term commitment and contributions to the study.

\section{CONFLICT OF INTEREST}

The authors do not have any disclosures to report. The views expressed in this manuscript are those of the authors and do not necessarily represent the views of the National Institutes of Health; or the US Department of Health and Human Services.

\section{ORCID}

Lisa B. VanWagner (iD http://orcid.org/0000-0002-6264-2573

\section{REFERENCES}

1. Vernon G, Baranova A, Younossi ZM. Systematic review: the epidemiology and natural history of non-alcoholic fatty liver disease and non-alcoholic steatohepatitis in adults. Aliment Pharmacol Ther. 2011;34:274-285.

2. Feldstein $A E$, Charatcharoenwitthaya $P$, Treeprasertsuk $S$, Benson JT, Enders FB, Angulo P. The natural history of non-alcoholic fatty liver disease in children: a follow-up study for up to 20 years. Gut. 2009;58:1538-1544.
3. Rinella ME. Nonalcoholic fatty liver disease: a systematic review. JAMA. 2015;313:2263-2273.

4. Ascha MS, Hanouneh IA, Lopez R, Tamimi TA, Feldstein AF, Zein NN. The incidence and risk factors of hepatocellular carcinoma in patients with nonalcoholic steatohepatitis. Hepatology. 2010;51:1972-1978.

5. Charlton MR, Burns JM, Pedersen RA, Watt KD, Heimbach JK, Dierkhising RA. Frequency and outcomes of liver transplantation for nonalcoholic steatohepatitis in the United States. Gastroenterology. 2011;141:1249-1253.

6. Targher G, Day CP, Bonora E. Risk of cardiovascular disease in patients with nonalcoholic fatty liver disease. $N$ Engl J Med. 2010;363:1341-1350.

7. Soderberg C, Stal P, Askling J, et al. Decreased survival of subjects with elevated liver function tests during a 28-year follow-up. Hepatology. 2010;51:595-602.

8. Browning JD, Szczepaniak LS, Dobbins R, et al. Prevalence of hepatic steatosis in an urban population in the United States: impact of ethnicity. Hepatology. 2004;40:1387-1395.

9. Colditz GA, Willett WC, Rotnitzky A, Manson JE. Weight gain as a risk factor for clinical diabetes mellitus in women. Ann Intern Med. 1995;122:481-486.

10. Willett WC, Manson JE, Stampfer MJ, et al. Weight, weight change, and coronary heart disease in women. Risk within the 'normal' weight range. JAMA. 1995;273:461-465.

11. Rexrode KM, Hennekens $\mathrm{CH}$, Willett WC, et al. A prospective study of body mass index, weight change, and risk of stroke in women. JAMA. 1997;277:1539-1545

12. Wakasugi $\mathrm{M}$, Narita I, Iseki K, et al. Weight gain after 20 years of age is associated with prevalence of chronic kidney disease. Clin Exp Nephrol. 2012;16:259-268.

13. Du S, Wang C, Jiang W, et al. The impact of body weight gain on nonalcoholic fatty liver disease and metabolic syndrome during earlier and later adulthood. Diabetes Res Clin Pract. 2016;116:183-191.

14. Kimura T, Deshpande GA, Urayama KY, Masuda K, Fukui T, Matsuyama Y. Association of weight gain since age 20 with non-alcoholic fatty liver disease in normal weight individuals. J Gastroenterol Hepatol. 2015;30:909-917.

15. Friedman GD, Cutter GR, Donahue RP, et al. CARDIA: study design, recruitment, and some characteristics of the examined subjects. J Clin Epidemiol. 1988;41:1105-1116.

16. Chalasani N, Younossi Z, Lavine JE, et al. The diagnosis and management of non-alcoholic fatty liver disease: practice Guideline by the American Association for the Study of Liver Diseases, American College of Gastroenterology, and the American Gastroenterological Association. Hepatology. 2012;55:2005-2023.

17. Parker ED, Schmitz KH, Jacobs DR, Dengel DR, Schreiner PJ. Physical activity in young adults and incident hypertension over 15 years of follow-up: the CARDIA study. Am J Public Health. 2007;97:703-709.

18. Mertens I, Van Gaal LF. New International Diabetes Federation (IDF) and National Cholesterol Education Program Adult Treatment panel III (NCEP-ATPIII) criteria and the involvement of hemostasis and fibrinolysis in the metabolic syndrome. J Thromb Haemost. 2006;4:1164-1166.

19. VanWagner LB, Ning H, Lewis CE, et al. Associations between nonalcoholic fatty liver disease and subclinical atherosclerosis in middleaged adults: the Coronary Artery Risk Development in Young Adults Study. Atherosclerosis. 2014;235:599-605.

20. Kodama Y, Ng CS, Wu TT, et al. Comparison of CT methods for determining the fat content of the liver. AJR Am J Roentgenol. 2007;188:1307-1312

21. Nagin DS, Odgers CL. Group-based trajectory modeling (nearly) two decades later. J Quant Criminol. 2010;26:445-453.

22. Allen NB, Siddique J, Wilkins JT, et al. Blood pressure trajectories in early adulthood and subclinical atherosclerosis in middle age. JAMA. 2014:311:490-497. 
23. Montonen J, Boeing H, Schleicher E, Fritsche A, Pischon T. Association of changes in body mass index during earlier adulthood and later adulthood with circulating obesity biomarker concentrations in middle-aged men and women. Diabetologia. 2011;54:1676-1683.

24. Weston SR, Leyden W, Murphy R, et al. Racial and ethnic distribution of nonalcoholic fatty liver in persons with newly diagnosed chronic liver disease. Hepatology. 2005;41:372-379.

25. Musso G, Gambino R, Cassader M, Pagano G. Meta-analysis: natural history of non-alcoholic fatty liver disease (NAFLD) and diagnostic accuracy of non-invasive tests for liver disease severity. Ann Med. 2011;43:617-649.

26. Mofrad P, Contos MJ, Haque M, et al. Clinical and histologic spectrum of nonalcoholic fatty liver disease associated with normal ALT values. Hepatology. 2003;37:1286-1292.

27. Blais P, Husain N, Kramer JR, Kowalkowski M, El-Serag H, Kanwal F. Nonalcoholic fatty liver disease is underrecognized in the primary care setting. Am J Gastroenterol. 2015;110:10-14.

28. Cazzo E, Jimenez LS, Pareja JC, Chaim EA. Effect of Roux-en-Y gastric bypass on nonalcoholic fatty liver disease evaluated through NAFLD fibrosis score: a prospective study. Obes Surg. 2015;25: 982-985.

29. Winder JS, Dudeck BS, Schock S, Lyn-Sue JR, Haluck RS, Rogers AM. Radiographic improvement of hepatic steatosis after laparoscopic Roux-en-Y gastric bypass. Obes Surg. 2017;27:376-380.

30. Zhang HJ, He J, Pan LL, et al. Effects of moderate and vigorous exercise on nonalcoholic fatty liver disease: a randomized clinical trial. JAMA Intern Med. 2016;176:1074-1082.

31. Vilar-Gomez E, Martinez-Perez Y, Calzadilla-Bertot L, et al. Weight loss through lifestyle modification significantly reduces features of nonalcoholic steatohepatitis. Gastroenterology. 2015;149:367-378. e365; quiz e314-365.

32. Lassailly G, Caiazzo R, Buob D, et al. Bariatric surgery reduces features of nonalcoholic steatohepatitis in morbidly obese patients. Gastroenterology. 2015;149:379-388 ; quiz e315-376.

33. Wing RR, Phelan S. Long-term weight loss maintenance. Am J Clin Nutr. 2005;82(1 Suppl):222s-225s.

34. Promrat K, Kleiner DE, Niemeier HM, et al. Randomized controlled trial testing the effects of weight loss on nonalcoholic steatohepatitis. Hepatology. 2010;51:121-129.

35. Hanson MA, Gluckman PD, Ma RC, Matzen P, Biesma RG. Early life opportunities for prevention of diabetes in low and middle income countries. BMC Public Health. 2012;12:1025.

\section{SUPPORTING INFORMATION}

Additional Supporting Information may be found online in the supporting information tab for this article.

How to cite this article: VanWagner LB, Khan SS, Ning H, et al. Body mass index trajectories in young adulthood predict non-alcoholic fatty liver disease in middle age: The CARDIA cohort study. Liver Int. 2018;38:706-714. https://doi. org/10.1111/liv.13603 